\title{
Erhöhtes Osteoporoserisiko
}

\section{Bei Darm-Krankheiten die Knochen nicht vergessen!}

\author{
Nicht nur Darmerkrankungen selbst, sondern auch ihre Therapie können einen ungünstigen \\ Einfluss auf die Knochendichte entfalten. Bei welchen Patienten sollte man nach einer ernied- \\ rigten Knochendichte fahnden und evtl. eine Behandlung einleiten?
}

_ Calciferole, sprich Vitamin D, werden mit den Nahrungsfetten im Zwölffingerdarm durch Gallensäuren in Mizellen zusammengefasst und dann resorbiert. Die Kalziumaufnahme erfolgt ebenfalls im Dünndarm als aktiver, von Vitamin D abhängiger Transport. Diese Mechanismen sind bei vielen Magen-DarmKrankheiten gestört mit der Folge eines erhöhten Osteoporoserisikos, erklärte Prof. I. A. Harsch, Saalfelden. So hat eine Magenresektion eine ungenügende Nahrungsausnutzung zur Folge. Es kommt zu einer unzureichenden Stimulation des Pankreas, und der Speisebrei wird nicht ausreichend mit Galle vermischt. Folge ist eine verminderte Resorption von Kalzium und Vitamin D.

\section{Bei Zöliakie und CED häufig}

Besonders häufig kommt es bei Zöliakiepatienten zur Osteoporose, was auch mit der oft verspäteten Diagnosestellung zu tun hat. Die chronische Dünndarmentzündung beeinträchtigt die Kalziumund Vitamin-D-Aufnahme.

„Auch bei Patienten mit einer chronisch-entzündlichen Darmerkrankung (CED) tritt eine Osteopenie bzw. Osteoporose gehäuft auf“, erläuterte Harsch. Pathogenetisch spielt hier neben der Vitamin-D- und Kalzium-Aufnahmestörung auch der Inflammationsprozess eine wichtige Rolle. Die bei diesen Erkrankungen vermehrt gebildeten Zytokine aktivieren die Osteoklasten und hemmen die Osteoblasten. Das Risiko für Wirbel- und Hüftfrakturen ist bei CED-Patienten deutlich erhöht. Deshalb

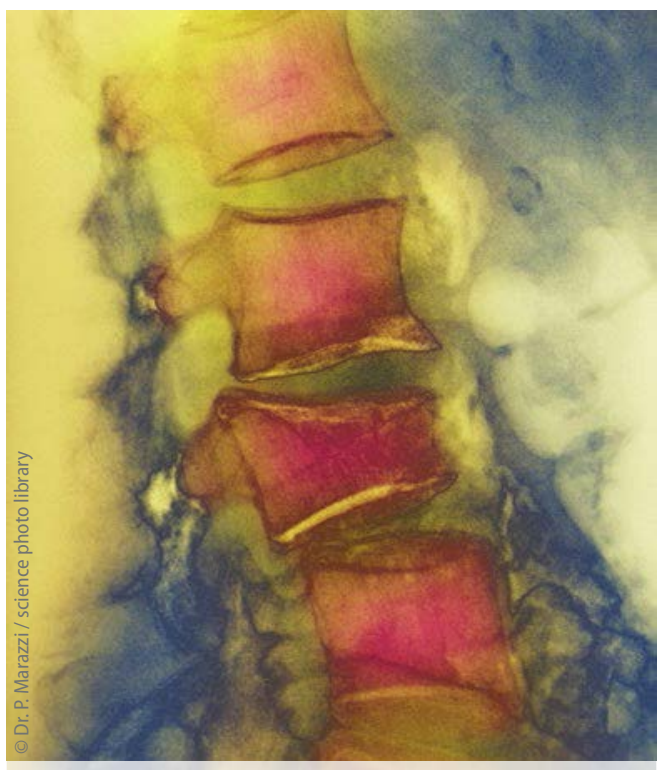

Kommt der Knochenschwund vom Darm?

wird in der Leitlinie empfohlen, bei erwachsenen CED-Patienten, die längerfristig systemisch Steroide erhalten oder einen chronisch aktiven Krankheitsverlauf zeigen, mittels DXA-Messung die Knochendichte an der LWS und am proximalen Femur zu bestimmen.

\section{Einflüsse von Medikamenten}

Neben Kortikosteroiden fördern noch weitere Medikamente, die zur Therapie gastroenterologischer Erkrankungen verschrieben werden, das Osteoporoserisiko. So steigt bereits nach einjähriger Einnahme von Protonen-Pumpen-Hemmern (PPI) das Risiko auf das 1,22-Fache und nach 4 Jahren um den Faktor 1,59. „Warum das so ist, weiß man noch nicht genau, aber für die optimale Kalziumresorption scheint wohl das Vorhandensein von Magensäure entscheidend zu sein“, so Harsch.

\section{An Vitamin D denken}

Bei Patienten mit Darmerkrankungen sind grundsätzlich für die Prävention bzw. Therapie der Osteoporose dieselben Basismaßnahmen zu empfehlen wie bei Darmgesunden. Dazu gehören Sport, nur mäßiger Alkoholkonsum, Einstellen des Rauchens und der Versuch, eine Normalisierung der Kalzium- und VitaminD-Aufnahme sicherzustellen, was bei Patienten mit einer Darmerkrankung nicht immer einfach ist. Da Phosphat die Verfügbarkeit von Kalzium verringert, sollte die Phosphataufnahme gedrosselt werden. Phosphatreich sind Fleisch- und Wurstwaren, Schmelzkäse, Bier und Cola.

Und wie sieht es mit einer Vitamin-D-Substitution aus? „Ein Screening auf Vitamin-D-Mangel ist bei der gegenwärtigen Datenlage nur dann angezeigt, wenn aufgrund einer Risikosituation oder des klinischen Verdachts ein schwerer Mangelzustand mit Gefahr einer Osteomalazie befürchtet wird“, so Harsch. Dies gilt für Patienten mit einer Zöliakie oder CED.

Bei CED sollte die Dosierung des Steroids soweit wie möglich heruntergefahren bzw. die Therapie auf ein Biologikum umgestellt werden. Bei Nachweis einer Osteoporose besteht die Indikation für ein Bisphosphonat. Zugelassen für die Steroidosteoporose sind $5 \mathrm{mg} \mathrm{Zo-}$ ledronsäure einmal jährlich, $20 \mu \mathrm{g} / \mathrm{Tag}$ Teriparatid, $10 \mathrm{mg} / \mathrm{Tag}$ Alendronsäure und $5 \mathrm{mg} /$ Tag Risedronsäure.

\section{Dr. Peter Stiefelhagen}

- 52. Ärztekongress der Bezirksärztekammer Nordwürttemberg, 27.1.2019 in Stuttgart 